Pesq. Vet. Bras. 36(Supl.1):65-70, junho 2016 DOI: $10.1590 / \mathrm{S} 0100-736 \mathrm{X} 2016001300010$

\title{
Valores hematológicos e bioquímicos de pinguins-de-Magalhães em reabilitação no Espírito Santo, sudeste do Brasil ${ }^{1}$
}

\author{
Luis F.S.P. Mayorga ${ }^{2,3 *}$, Renata C.C. Bhering ${ }^{2}$, Laila C.C. Medeiros ${ }^{2,3}$, Eduardo L.F. Silva ${ }^{2,3}$, \\ Yhuri C. Nóbrega ${ }^{4}$, Maria C.V. Rangel ${ }^{3}$, Leandro A. Fonseca ${ }^{5}$ e João L. Rossi Junior ${ }^{3}$
}

\begin{abstract}
Mayorga L.F.S.P., Bhering R.C.C., Medeiros L.C.C., Silva E.L.F., Nóbrega Y.C., Rangel M.C.V., Fonseca L.A. \& Rossi Junior J.L. 2016. [Hematological and biochemical values of Magellanic penguins in rehabilitation in Espírito Santo, southeastern Brazil.] Valores hematológicos e bioquímicos de pinguins-de-Magalhães em reabilitação no Espírito Santo, sudeste do Brasil. Pesquisa Veterinária Brasileira 36(Supl.1):65-70. Instituto de Pesquisa e Reabilitação de Animais Marinhos, Rodovia BR-262 Km 0, Cariacica, ES 29140-130, Brazil. E-mail: luisfelipe@ipram-es.org.br

This paper reports hematological and biochemical values of juveniles Magellanic penguins (Spheniscus magellanicus) stranded in Espírito Santo and Rio de Janeiro, Southeastern Brazil, that were sent for rehabilitation. The average values were: erythrocytes $2.55 \pm 0,54$ milhões $/ \mu \mathrm{L}$, hemoglobin $13.37 \pm 3,91 \mathrm{~g} / \mathrm{dL}$, hematocrit $41.5 \pm 0.04 \%$, plasma protein $6.34 \pm 0.81 \mathrm{~g} / \mathrm{dL}$, leukocytes $16,301 \pm 6,402 / \mu \mathrm{L}$, thrombocytes $20,516 \pm 4,591$ células/ $\mu \mathrm{L}^{3}$, mean cell volume (MCV) $172.0 \pm 53.0 \mathrm{fL}$, mean cell hemoglobin concentration (MCHC) $26.41 \pm 0.02 \%$, alanine transaminase (ALT) $50.0 \pm 17.89 \mathrm{U} / \mathrm{L}$, uric acid $8.93 \pm 3.0 \mathrm{mg} / \mathrm{dL}$, albu$\min 1.68 \pm 0.53 \mathrm{mg} / \mathrm{dL}$, calcium $9.7 \pm 0.57 \mathrm{mg} / \mathrm{dL}$, phosphorus $10.39 \pm 8.5 \mathrm{mg} / \mathrm{dL}$ and glucose $211.6 \pm 30.3 \mathrm{mg} / \mathrm{dL}$. Values of erythrocytes, MCV and MCH were discrepant in relation to the current reference values. Leukocytes and thrombocytes values has meant inconclusive. Hematocrit, hemoglobin, plasma protein and biochemical values provided important contribution for the establishment of reference parameters.
\end{abstract}

INDEX TERMS: Seabirds, blood, hematology, hematology parameters, Magellanic penguins, Spheniscus magellanicus, Speniscidae, juveniles.

RESUMO.- Este trabalho apresenta valores hematológicos e bioquímicos de pinguins-de-Magalhães (Spheniscus magellanicus) juvenis que arribaram no Espírito Santo e Rio de Janeiro, litoral sudeste do Brasil, e foram encaminhados a reabilitação. Os valores médios obtidos foram: eritrócitos $2.55 \pm 0.54$ milhões/ $\mu \mathrm{L}$; hemoglobina $13,37 \pm 3,91 \mathrm{~g} / \mathrm{dL}$; hematócrito 41,5 $\pm 0,04 \%$; proteína plasmática $6,34 \pm 0,81 \mathrm{~g} / \mathrm{dL}$; leucócitos $16.301 \pm 6.402 / \mu \mathrm{L}$; trombócitos $20.516 \pm 4.591$ células/ $\mu \mathrm{L}$; volume corpuscular médio (VCM) $172,0 \pm 53,0 \mathrm{fL}$;

\footnotetext{
${ }^{1}$ Recebido em 1 de junho de 2015.

Aceito para publicação em 13 de março de 2016.

${ }^{2}$ Instituto de Pesquisa e Reabilitação de Animais Marinhos, Rodovia BR$262 \mathrm{Km} \mathrm{0,} \mathrm{Cariacica,} \mathrm{ES} \mathrm{29140-130,} \mathrm{Brasil.} \mathrm{*Autor} \mathrm{para} \mathrm{correspondência:}$ luisfelipe@ipram-es.org.br

${ }^{3}$ Universidade de Vila Velha (UVV), Av. Comissário José Dantas de Melo 21, Vila Velha, ES 29102-920, Brasil.

${ }^{4}$ Instituto de Ensino, Pesquisa e Preservação Ambiental Marcos Daniel, Rua Fortunato Ramos 123, Vitória, ES 29055-290, Brasil.

${ }^{5}$ Universidade Federal de Viçosa (UFV), Avenida PH Rolfs s/n, Viçosa, MG 36570-900, Brasil.
}

concentração de hemoglobina corpuscular média (CHCM) 26,41 $\pm 0,02 \%$; alanina transaminase (ALT) 50,0 $\pm 17,89$ U/L; ácido úrico 8,93 $\pm 3,0 \mathrm{mg} / \mathrm{dL}$; albumina $1,68 \pm 0,53 \mathrm{~g} / \mathrm{dL}$; cálcio $9,7 \pm 0,57 \mathrm{mg} / \mathrm{dL}$; fósforo $10,39 \pm 8,5 \mathrm{mg} / \mathrm{dL}$ e glicose $211,6 \pm 30,3 \mathrm{mg} / \mathrm{dL}$. Valores de eritrócitos, VCM e CHCM foram discrepantes em relação aos valores de referência atuais. Valores de leucócitos e trombócitos tem significado inconclusivo. Hematócrito, hemoglobina, proteína plasmática e valores bioquímicos forneceram importante contribuição para o estabelecimento de parâmetros de referência.

TERMOS DE INDEXAÇÃO: Aves marinhas, sangue, hematologia, parâmetros hematológicos, pinguins-de-Magalhães, Spheniscus magellanicus, Spheniscidae, juvenis.

\section{INTRODUÇÃO}

Os pinguins-de-Magalhães (Spheniscus magellanicus Forster, 1781) podem ser encontrados comumente na região sul do Brasil, onde a ocorrência de arribamentos, resgate e reabilitação muitas vezes estão relacionados ao contex- 
to de contaminação por óleo e derivados no mar (Barbosa 2003, García-Borboroglu et al. 2006, Silva Filho \& Ruoppolo 2006, García-Borboroglu et al. 2010, Mäder, Sander \& Casa Junior 2010). Relatos informais sobre a ocorrência esporádica de alguns pinguins-de-Magalhães no sul do estado do Espírito Santo (ES) são comuns, sendo este fenômeno conhecido por algumas comunidades litorâneas há algumas décadas, quando essas aves costumavam aparecer em baixa quantidade nos meses de inverno. Entretanto, a partir da primeira década do século XXI pinguins-de-Magalhães juvenis passaram a arribar em massa e sazonalmente no ES e em unidades federativas próximas tais como Rio de Janeiro e Bahia, demandando o desenvolvimento de atividades de reabilitação e soltura (Silva et al. 2001, Pontes et al. 2007, García-Borboroglu et al. 2010).

Pinguins são aves pelágicas especializadas para o nado e o mergulho, possuindo rantoteca fina e longa, adaptada ao seu hábito alimentar. Possuem corpo fusiforme com pescoço e cauda curtos; pernas com tibiotarso curto, três dedos palmípedes e um dedo vestigial. Os ossos são densos, sólidos e não-pneumáticos, e os membros torácicos foram modificados em nadadeiras (Silva Filho \& Ruoppolo 2015). Os pinguins-de-Magalhães alcançam os $70 \mathrm{~cm}$ de altura e pesam de três a quatro quilos e meio (Williams 1995, Sick 1997, Silva Filho \& Ruppolo 2015). A temperatura corporal varia entre 39 e 41 graus Celsius $\left({ }^{\circ} \mathrm{C}\right)$, e o gênero Spheniscus tolera temperaturas entre 0 e $30^{\circ} \mathrm{C}$. Atingem a maturidade reprodutiva entre quatro e sete anos. A temporada reprodutiva ocorre nos meses de verão, nas costas dos oceanos Atlântico $\left(42-55^{\circ} \mathrm{S}\right)$ e Pacífico

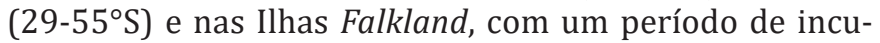
bação de 40 a 45 dias. Macho e fêmea dividem os cuidados parentais, que duram entre oito e 16 semanas (Fowler \& Cubas 2001, Silva Filho \& Ruoppolo 2015). A dieta é composta por sardinha (Sprattus fuegensis Jenyns, 1842), merluza (Merluccius hubbsi Marini, 1933) e principalmente por anchoíta (Engraulis anchoita Hubbs et Marini, 1935), que pode compor até $72 \%$ de sua dieta. As espécies predadas variam ao longo da distribuição geográfica das colônias de pinguins, e diversas espécies de moluscos cefalópodes (lulas e polvos) podem ser incluídas em sua dieta quando há escassez de peixes (Frere et al. 1996, Silva Filho \& Ruoppolo 2015). A maior colônia de pinguins-de-Magalhães localiza-se em Punta Tombo, Argentina, e sua população está em declínio (Silva Filho \& Ruoppolo 2015, Boersma 2008), entretanto a espécie ainda é considerada como Quase Ameaçada - Near Threatened - na Lista Vermelha de Espécies Ameaçadas da IUCN (BirdLife International 2012).

Após a fase de muda, aproximadamente no fim do mês de fevereiro, inicia-se o período migratório anual (Pütz, Ingham \& Smith 2000, Pütz et al. 2007), e na costa brasileira os pinguins juvenis podem ser vistos a partir de junho, sendo comum o registro dessas aves no litoral do Rio Grande do Sul e de Santa Catarina (Sick 1997, Petry \& Fonseca 2002, Silva Filho \& Ruoppolo 2015, Pinto et al. 2007, Mäder et al. 2010). A ocorrência da espécie parece ser normal até $23^{\circ} \mathrm{S}$ na costa do Brasil, o que equivale ao litoral do RJ (Del Hoyo et al. 1992, Sick 1997, Stokes et al. 1998).
No contexto da reabilitação de pinguins arribados nas praias, a análise de parâmetros hematológicos é indispensável ao se avaliar o estado de saúde individual e optar por devolver ou não um animal à vida livre. Entretanto, não existem valores hematológicos ou bioquímicos bem estabelecidos para pinguins (Silva Filho \& Ruoppolo 2015). Moreno et al. (2002) relataram valores de hematócrito para pinguins-de-Magalhães adultos de vida livre em estação reprodutiva, 47 machos e 53 fêmeas. Campos et al. (2008) relataram valores hematológicos de nove pinguins-de-Magalhães mantidos em cativeiro em um zoológico, porém, perceberam que grande parte dos pinguins apresentava alterações tais quais desidratação, distúrbios gastrintestinais, distrição respiratória e baixo peso, o que provavelmente influenciou no resultado dos exames. Posteriormente, Campos (2011) apresentou valores hematológicos de dois grupos de pinguins-de-Magalhães; positivos ou negativos para infecção com malária aviária, 75 aves no total. Coraiola (2012) apresentou valores hematológicos e bioquímicos de mais de 30 pinguins-de-Magalhães juvenis e adultos em cativeiro. Almeida et al. (2007) relataram que a glicemia sérica de 21 pinguins-de-Magalhães juvenis mantidos em cativeiro variou entre 182 e $222 \mathrm{mg} / \mathrm{dl}$, enquanto Medeiros et al. (2011) relataram uma glicemia média de $190 \pm 4,45 \mathrm{mg} / \mathrm{dl}$ em 36 pinguins-de-Magalhães juvenis em atividade de reabilitação e soltura. Silva Filho \& Ruoppolo (2015) relatam valores hematológicos do pinguim-de-Magalhães em cativeiro no Brasil para referência, compilando 67 exames de 50 animais. Thrall (2015) considera que são necessárias no mínimo 40 amostras para se definir valores de referência, mas que para se obter precisão aceitável na definição dos valores de referência devem ser examinados no mínimo 120 animais.

Na África do Sul também se observa dificuldades em estabelecer parâmetros para o pinguim-africano (Spheniscus demersus Linnaeus, 1758), de maneira que um estudo realizou análise de valores hematológicos de mais de cem animais e comparou os valores com estudos anteriores (Parsons et al. 2015). De forma semelhante, o presente trabalho apresenta a média de alguns valores hematológicos de pinguins-de-Magalhães juvenis que arribaram no litoral do Espírito Santo (ES) e Rio de Janeiro (RJ) no ano de 2011, mantidos em cativeiro para reabilitação no ES, como objetivo de contribuir com a formação de banco de dados de valores hematológicos de pinguins-de-Magalhães agrupados em um grupo homogêneo - juvenis em sua primeira migração de inverno - para aplicação em atividades que envolvam o manejo dessas aves em cativeiro.

\section{MATERIAL E MÉTODOS}

Procurou-se obter valores hematológicos de pinguins-de-magalhães que arribaram em estado de grave debilidade no litoral do Espírito Santo e do Rio de Janeiro no ano de 2011. A maior parte dos pinguins examinados era juvenil com menos de um ano de idade em sua primeira migração invernal; apenas três pinguins eram juvenis remanescentes do inverno anterior. Todos os pinguins deram entrada com baixo peso e escore corporal caquético ou magro, evidenciando os contornos ósseos. Dentre os 34 pinguins, $19(55,8 \%)$ haviam sido afetados por variados graus de contaminação por óleo quando em vida livre. As aves foram mantidas em 
cativeiro para reabilitação e posterior soltura sob responsabilidade do Instituto de Pesquisa e Reabilitação de Animais Marinhos (IPRAM), no ES. Quando os exames sanguíneos ocorreram a maior parte dos pinguins já estava em adiantado estágio de reabilitação, apresentando comportamento ativo, apetite e ganho de peso; e as aves sujas de óleo já haviam sido descontaminadas conforme orientado por Silva Filho \& Ruoppolo (2015). As aves eram alimentadas duas vezes ao dia, pela manhã e pela tarde. Ao longo do segundo semestre foram realizadas cinco campanhas de colheita de sangue, sempre no início da manhã, de forma que quando as colheitas ocorriam, os pinguins estavam em jejum hídrico e alimentar de aproximadamente 12 horas. Por meio de contenção física, foram coletados aproximadamente $2 \mathrm{ml}$ de sangue dos vasos tibiotársicos por gotejamento, utilizando-se agulhas hipodérmicas 25x7 e 25x8 descartáveis (Fig.1). 0 sangue foi armazenado em tubos com heparina e sem anticoagulante, e processado no mesmo dia no Laboratório Clínico do Hospital Veterinário Prof. Ricardo Alexandre Hippler da Universidade Vila Velha (UVV). 0 hematócrito foi realizado manualmente de acordo com a técnica de microhematócrito descrita por Hendrix (2005); a concentração de hemoglobina, pelo método da cianometahemoglobina, através de kit comercial (Bioclin Quibasa, Química Básica Ltda. - Belo Horizonte, MG, Brasil). As contagens de eritrócitos e leucócitos foram realizadas em câmara de Neubauer, tendo como diluente a solução de Natt \& Herrick (1952). As contagens diferenciais de leu-

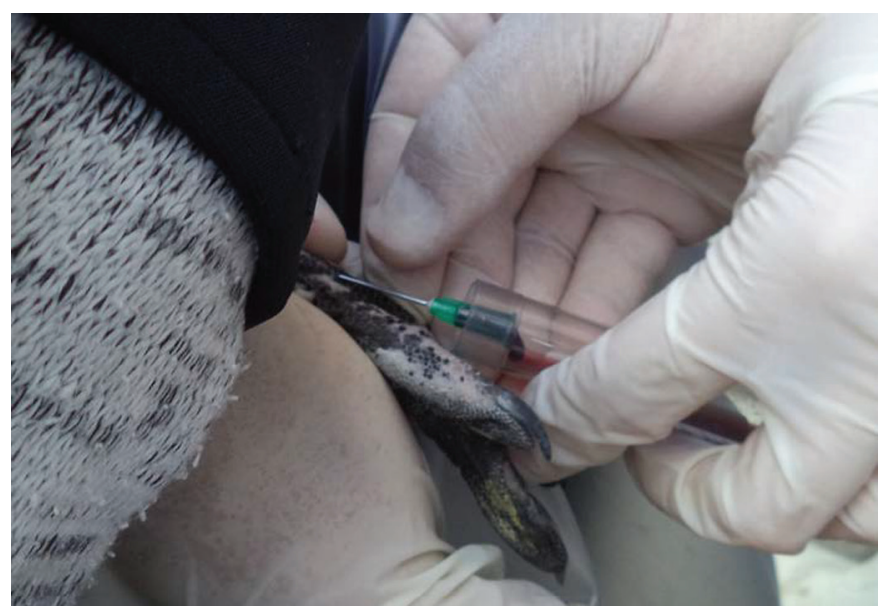

Fig.1. Colheita de sangue por gotejamento dos vasos tibiotársicos em pinguim-de-Magalhães (Spheniscus magellanicus). cócitos e de trombócitos foram estabelecidas em esfregaço sanguíneo corado segundo a técnica de Rosenfeld descrita por Thrall (2015). A partir das variáveis do eritrograma estabeleceram-se matematicamente os índices hematimétricos VCM e CHCM. A glicemia foi determinada através de glicosimetro digital portátil Accu-Chek ${ }^{\circledR}$. Para avaliação dos dados obtidos determinou-se média, desvio padrão, erro padrão e o intervalo de confiança associada. Para o cálculo do intervalo de confiança, utilizou-se um grau de confiança de 95\%. Para comparação dos valores, utilizaram-se os parâmetros hematológicos de pinguins-de-Magalhães em cativeiro no Brasil e de outras espécies do mesmo gênero, tais como o pinguim-de-Humboldt (Spheniscus humboldti Meyen, 1834) e o pinguim-africano (Spheniscus demersus) (Silva Filho \& Ruoppolo 2015). Os valores hematológicos para S. magellanicus apresentados por outros autores (Moreno et al. 2002, Almeida et al. 2007, Campos 2011, Medeiros et al. 2011, Coraiola 2012) não foram utilizados como referência devido ao baixo número amostral, porém foram utilizados para comparação em alguns casos. Os pinguins examinados não foram sexados. A pesquisa foi aprovada pela Comissão de Ética no Uso de Animais da Universidade Vila Velha (CEUA-UVV) (Registro da CEUA: 139/2010) e o IPRAM foi autorizado a realizar colheita e transporte de amostras biológicas, pesquisa e manutenção dos pinguins em cativeiro (SISBIO 26896).

\section{RESULTADOS}

Foram realizados exames hematológicos para avaliação de eritrócitos, hemoglobina, hematócrito, proteína plasmática, leucócitos totais e trombócitos em 34 pinguins; 47 exames no total, considerando que 13 indivíduos repetiram esses exames (Quadro 1). Para contagem de eritrócitos obteve-se média de 2,55 $\pm 0,54$ milhões/ $\mu \mathrm{L}$, valores acima do limite superior de referência descritos por Silva Filho \& Ruoppolo (2015). A concentração de hemoglobina obteve

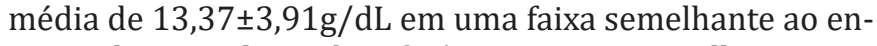
contrado nos valores de referência para $S$. magellanicus e $S$. humboldti (Silva Filho \& Ruoppolo 2015). Para o hemató-

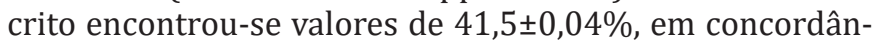
cia com a referência (Silva Filho \& Ruoppolo 2015). Para proteína plasmática obteve-se a média de $6,34 \pm 0,81 \mathrm{~g} / \mathrm{dL}$, mais alta que a faixa de normalidade de referência (Silva Filho \& Ruoppolo 2015). Para leucócitos obteve-se a média de $16.301 \pm 6.402 / \mu \mathrm{L}$, valor mais alto que a referência (Silva Filho \& Ruoppolo 2015). Para trombócitos obteve-se a mé-

Quadro 1. Valores hematológicos de Spheniscus magellanicus juvenis originários de vida livre, mantidos em cativeiro para reabilitação

\begin{tabular}{|c|c|c|c|c|c|c|c|}
\hline Parâmetro & $\mathrm{N}$ & Média & $\begin{array}{l}\text { Desvio } \\
\text { padrão }\end{array}$ & $\begin{array}{c}\text { Erro } \\
\text { padrão }\end{array}$ & $\begin{array}{c}\text { Intervalo de } \\
\text { confiança }\end{array}$ & Referência* & Comentários \\
\hline Eritrócitos (milhões/ $\mu \mathrm{L}$ ) & 47 & 2,55 & 0,54 & 0,27 & $2,39-2,70$ & $1,79 \pm 0,31$ & Discrepante \\
\hline Hemoglobina (g/dL) & 47 & 13,37 & 3,91 & 1,95 & $12,22-14,51$ & $13,47 \pm 1,55$ & Compatível \\
\hline Hematócrito (\%) & 47 & 41,50 & 0,04 & 0,02 & $41,48-41,51$ & $40,81 \pm 4,7$ & Compatível \\
\hline VCM (fL) & 27 & 172,0 & 53,0 & 26,5 & $151,03-192,96$ & $231,9 \pm 36,3$ & Discrepante \\
\hline CHCM (\%) & 27 & 26,41 & 0,02 & 0,01 & $26,40-26,41$ & $32,8 \pm 2,4$ & Discrepante \\
\hline Leucócitos $(103 / \mu \mathrm{L})$ & 47 & 16,30 & 6,40 & 3,20 & $14,42-18,17$ & $12,170 \pm 5,036$ & Discrepante \\
\hline Trombócitos $(/ \mu \mathrm{L})$ & 47 & 20.516 & 4.591 & 2.295 & $19.1680-21.863$ & - & Sem Referência \\
\hline Proteína Plasmática (g/dL) & 47 & 6,34 & 0,81 & 0,40 & $6,10-6,57$ & $5,1 \pm 0,9$ & Compatível \\
\hline Albumina (g/dL) & 23 & 1,68 & 0,53 & 0,26 & $1,45-1,90$ & $1,8 \pm 0,6$ & Compatível \\
\hline $\operatorname{ALT}(\mathrm{U} / \mathrm{L})$ & 23 & 50,02 & 17,89 & 8,94 & $42,28-57,75$ & - & Sem Referência \\
\hline Ácido Úrico (mg/dL) & 23 & 8,93 & 3,00 & 1,50 & $7,63-10,22$ & $5,21 \pm 2,8$ & Compatível \\
\hline Cálcio (mg/dL) & 23 & 9,70 & 0,57 & 0,28 & $9,45-9,94$ & - & Sem Referência \\
\hline Fósforo (mg/dL) & 23 & 3,16 & 1,58 & 0,79 & $2,47-3,84$ & - & Sem Referência \\
\hline Glicose (mg/dL) & 10 & 211,6 & 30,3 & 15,15 & $189,92-233,27$ & $224,2 \pm 63$ & Compatível \\
\hline
\end{tabular}

* Pinguins-de-Magalhães em cativeiro no Brasil; 50 animais, 67 exames (Silva Filho \& Ruoppolo 2015). 
dia de $20.516 \pm 4.591$ células $/ \mu \mathrm{L}$, sem valores de referência para comparação.

Foram calculados os índices hematimétricos volume corpuscular médio (VCM) e concentração de hemoglobina corpuscular média (CHCM) em 20 pinguins; 27 exames no total, considerando que sete indivíduos repetiram esses exames. Para VCM obteve-se a média de 172,0 $053,0 \mathrm{fL}$ e para CHCM obteve-se a média de $26,41 \pm 0,02 \%$, ambos apresentando valores abaixo do descrito por Silva Filho \& Ruoppolo (2015).

Foram realizados exames bioquímicos em 21 animais; 23 no total, considerando que dois indivíduos repetiram os exames. Para alanina aminotransferase (ALT) obteve-se a média de 50,02 $\pm 17,89 \mathrm{U} / \mathrm{L}$, sem valores de referência para comparação; sendo que $S$. demersus apresenta média de 38 U/L e Spheniscus humboldti apresenta média de $127 \pm 111$ U/L (Silva Filho \& Ruoppolo 2015). Para ácido úrico obteve-se a média de $8,93 \pm 3,0 \mathrm{mg} / \mathrm{dL}$, pouco acima do limite superior para a espécie; enquanto $S$. demersus apresenta média de $8,3 \pm 1,6 \mathrm{mg} / \mathrm{dL}$ e $S$. humboldti apresenta média de $11,2 \pm 8,2 \mathrm{mg} / \mathrm{dL}$ (Silva Filho \& Ruoppolo 2015). Para albumina obteve-se a média de $1,68 \pm 0,53 \mathrm{~g} / \mathrm{dL}$, semelhante à referência para a espécie; enquanto $S$. demersus apresenta média de $1,9 \pm 0,3 \mathrm{mg} / \mathrm{dL}$ e $S$. humboldti apresenta média de $2,1 \pm 0,6 \mathrm{mg} / \mathrm{dL}$ (Silva Filho \& Ruoppolo 2015). Para cálcio obteve-se a média de $9,7 \pm 0,57 \mathrm{mg} / \mathrm{dL}$ sem valores de referência para comparação; enquanto $S$. demersus apresenta média de $11,3 \pm 1,4 \mathrm{mg} / \mathrm{dL}$ e $S$. humboldti apresenta média de $10,7 \pm 2,3 \mathrm{mg} / \mathrm{dL}$ (Silva Filho \& Ruoppolo 2015). Para fósforo obteve-se a média de $3,16 \pm 1,58 \mathrm{mg} / \mathrm{dL}$, sem valores de referência para comparação; enquanto $S$. demersus apresenta média de $7,3 \pm 1,3 \mathrm{mg} / \mathrm{dL}$ e $S$. humboldti apresenta média de 3,9 $\pm 2,1 \mathrm{mg} / \mathrm{dL}$ (Silva Filho \& Ruoppolo 2015).

Para a glicemia de dez pinguins obteve-se a média de $211,6 \pm 30,3 \mathrm{mg} / \mathrm{dL}$, em uma faixa semelhante ao valor de referência (Silva Filho \& Ruoppolo 2015).

\section{DISCUSSÃO}

Pinguins juvenis e adultos em muda de penas podem apresentar perfis hematológicos distintos, com menores valores de hematócrito, hemoglobina e contagem de eritrócitos (Silva Filho \& Ruoppolo 2015). A contagem de eritrócitos e hematócrito em aves aumenta com a idade, e maiores valores são verificados em machos. A produção de eritropoietina aviária pelos rins aumenta em resposta a estímulos como hemorragia e hemólise, aumentando a produção de eritrócitos na medula óssea (Thrall 2015). No presente estudo, a contagem de eritrócitos acima da referência (Silva Filho \& Ruoppolo 2015) pode sugerir desidratação ou, uma adaptação dos animais às condições climáticas da região onde foram encontrados. No caso de desidratação, esta pode ser decorrente do processo crônico de subnutrição que levou os animais ao encalhe e do processo agudo de jejum ao qual, os animais foram submetidos na véspera, mas não se deve descartar possíveis falhas na contagem dos eritrócitos. Na interpretação de resultados hematológicos em pinguins-de-Magalhães, características morfológicas das células sanguíneas dificultam a distinção de alguns tipos celulares; mesmo patologistas clínicos experientes em hematologia aviária podem apresentar dificuldade nessa distinção (Silva Filho \& Ruoppolo 2015). Coraiola (2012), por exemplo, analisou dezenas de Spheniscus magellanicus e encontrou valor médio de eritrócitos dentro da faixa de normalidade da referência (Silva Filho \& Ruoppolo 2015). Como a contagem de eritrócitos influencia diretamente no cálculo dos índices hematimétricos, os valores de VCM e CHCM no presente trabalho estão abaixo da referência descrita por Silva Filho \& Ruoppolo (2015).

Nos eritrócitos das aves os compostos fosfatados que influenciam a afinidade da hemoglobina com o oxigênio são diferentes dos de mamíferos, de forma que tecidos das aves podem extrair oxigênio da hemoglobina mais facilmente. Valores de hemoglobina abaixo da referência poderiam sugerir anemia por desnutrição e deficiência de ferro (Thrall 2015). 0 valor médio de hemoglobina encontrado no presente estudo está muito semelhante à faixa de referência (Silva Filho \& Ruoppolo 2015). Além disso, Coraiola (2012) também encontrou valor médio de hemoglobina para S. magellanicus muito semelhante.

A análise do hematócrito tem grande importância na reabilitação de pinguins no Brasil, auxiliando na compreensão do quadro de saúde individual (Rodrigues et al. 2010). É o método mais rápido e prático de avaliação da massa eritrocitária de aves, e os valores normais para aves variam entre 35 e 55\% (Thrall 2015). Campos (2011) relata hematócrito de $37,18 \pm 8,24 \%$ para $S$. magellanicus negativos para malária aviária, e valor ainda menor para pinguins positivos. Os valores encontrados no presente trabalho estão dentro da faixa de normalidade (Silva Filho \& Ruoppolo 2015). 0 estudo de Moreno et al. (2002) com adultos em colônias reprodutivas e o estudo de Coraiola (2012) com juvenis e adultos após a reabilitação também encontraram valores médios de hematócrito para $S$. magellanicus compatíveis com a referência (Silva Filho \& Ruoppolo 2015).

O nível plasmático normal de proteínas é essencial para a manutenção da pressão osmótica coloidal fisiológica, que preserva o $\mathrm{pH}$ e o volume sanguíneo. Na maioria das aves, considera-se hiperproteinemia quando a concentração plasmática de proteína total é superior a $4,5 \mathrm{~g} / \mathrm{dL}$, sendo provocada por desidratação e/ou inflamação aguda ou crônica, dentre outros fatores (Thrall 2015). Apesar do valor de referência para o gênero Spheniscus ser em torno de $5 \mathrm{~g} /$ dL (Silva Filho \& Ruoppolo 2015), ou seja, ainda mais alto do que o relatado por Thrall (2015); o presente trabalho encontrou uma média de $6,34 \pm 0,81 \mathrm{~g} / \mathrm{dL}$. Para $S$. magellanicus Campos (2011) relata um valor médio de proteína plasmática menor, 4,08 $\pm 1,39 \mathrm{~g} / \mathrm{dL}$. Já Coraiola (2012) relata um valor médio de $5,8 \pm 0,8 \mathrm{~g} / \mathrm{dL}$. Possivelmente o alto valor obtido para proteína plasmática no presente trabalho deve-se a discreta desidratação decorrente do jejum, associada à utilização de uma dieta de ganho de peso durante a reabilitação.

Os leucócitos presentes no sangue das aves são linfócitos, monócitos e granulócitos, esses últimos classificados como heterófilos, eosinófilos e basófilos. O leucograma pode variar muito entre aves normais da mesma espécie. Como se estressam ao serem manipuladas, o processo de colheita do sangue pode resultar em leucocitose fisiológi- 
ca, devido ao aumento da quantidade de heterófilos e linfócitos no sangue periférico (Silva Filho \& Ruoppolo 2015, Thrall 2015). Além disso, pinguins debilitados com anemia regenerativa poderiam apresentar numerosos pró-eritroblastos confundíveis com linfócitos, que podem levar a um falso diagnóstico de leucocitose (Silva Filho \& Ruoppolo 2015). No presente trabalho a média de leucócitos estava acima da faixa de normalidade, assemelhando-se às médias encontradas por Campos (2011) e Coraiola (2012) para $S$. magellanicus. Segundo Thrall (2015) a faixa de valores de referência para leucócitos totais em aves é mais ampla que a de mamíferos domésticos, e os valores do leucograma de aves devem ser muito diferentes da faixa de referência para que tenham importância diagnóstica.

Trombócitos são células nucleadas no sangue periférico das aves, que participam no mecanismo hemostático da mesma forma que as plaquetas em mamíferos. Na maioria das espécies de aves a contagem de trombócitos varia de 20.000 a 30.000 células $/ \mu \mathrm{L}$, sendo difícil determinar valores verdadeiros devido à tendência dessas células em se aglomerar (Thrall 2015). Não foram encontrados valores de referência para trombócitos (Silva Filho \& Ruoppolo 2015), mas os valores obtidos no presente trabalho aparentemente encontram-se na faixa de normalidade de aves em geral (Thrall 2015) e são semelhantes aos resultados encontrados para S. magellanicus por Campos (2011).

No caso dos exames bioquímicos, são ainda mais escassos os parâmetros de referência de $S$. magellanicus, mas o método empregado permite assumir que os valores encontrados são confiáveis, sendo importantes para auxiliar na formação de valores de referência. A atividade plasmática da ALT, embora não seja a primeira escolha para detecção de alterações hepatocelulares em aves em geral, pode ser útil na investigação de doenças hepáticas (Thrall 2015).

Em aves, o ácido úrico é principal catabólito do metabolismo do nitrogênio, produzido no fígado e nos rins. Cerca de $90 \%$ do ácido úrico do sangue é removido pelos rins, de forma que a determinação do teor sérico ou plasmático desse ácido é utilizada para diagnóstico de doença renal em aves. Em geral, concentrações sanguíneas de ácido úrico acima de $15 \mathrm{mg} / \mathrm{dL}$ indicam alteração da função renal (Thrall 2015). Os valores encontrados no presente trabalho para ácido úrico estão discretamente superiores aos valores de referência para a espécie em cativeiro no Brasil, que é inferior aos valores de $S$. humboldti e $S$. demersus (Silva Filho \& Ruoppolo 2015), embora Villouta et al. (1997) tenham encontrado valores de ácido úrico de $S$. humboldti menores que os de $S$. magellanicus quando em vida livre. Da mesma forma, Campos (2011) relata um valor médio de ácido úrico mais elevado, semelhante ao encontrado no presente trabalho (principalmente em animais positivos para malária aviária). Já a pesquisa de Coraiola (2012) apresenta valores de ácido úrico muito elevados em S. magellanicus juvenis no período pré-reabilitação $(19,34 \pm 2,94 \mathrm{mg} / \mathrm{dL})$, que reduzem para juvenis em pós-reabilitação $(17,26 \pm 2,39$ e 9,36 $\pm 1,14 \mathrm{mg} / \mathrm{dL}$ ) e reduzem ainda mais em adultos pós-reabilitação $(7,88 \pm 0,73 \mathrm{mg} / \mathrm{dL})$, sendo a média total compatível com a referência (Silva Filho \& Ruoppolo 2015). Como os pinguins utilizados no presente estudo deram en- trada hipotérmicos, caquéticos e muito debilitados, podem ter sofrido algum grau de degeneração renal que justifique os valores mais elevados de ácido úrico encontrados.

A albumina representa de 40 a $50 \%$ do teor de proteína total no plasma de aves, sendo sintetizada no fígado. Em aves sadias o teor plasmático de albumina varia de 0,8 a $2 \mathrm{~g} / \mathrm{dL}$, embora esses valores possam não ser confiáveis (Thrall 2015). Os valores médios encontrados por Campos (2011), Coraiola (2012) e no presente trabalho aparentam normalidade, e são semelhantes ao valor médio de referência para albumina (Silva Filho \& Ruoppolo 2015).

Em aves o teor de cálcio total do plasma é influenciado pela concentração plasmática de albumina; existe correlação significativa entre o teor de albumina ou proteína total e o de cálcio total. Na maioria das aves não-poedeiras a concentração plasmática de cálcio varia de 8 a $11 \mathrm{mg} / \mathrm{dL}$ (Thrall 2015). Os valores encontrados no presente estudo estão dentro dessa faixa.

O teor plasmático de fósforo é controlado principalmente pela excreção renal. Em geral, aves jovens em crescimento tendem a fosfatemia mais elevada, se comparadas a aves adultas. Em aves considera-se hipofosfatemia valores abaixo de $5 \mathrm{mg} / \mathrm{dL}$ e hiperfosfatemia valores acima de $7 \mathrm{mg} / \mathrm{dL}$, essa última causada por doença renal grave (Thrall 2015). Apesar disso, se comparada com os valores de referência para S. humboldti (Silva Filho \& Ruoppolo 2015) a média encontrada no presente trabalho não indica hipofosfatemia, mas está dentro da faixa de normalidade.

Em frangos de corte a restrição alimentar moderada ou grave gera redução da concentração plasmática de proteínas e albumina, mas não afeta a concentração de glicose e fósforo (Rajman et al. 2006). A concentração sanguínea de glicose em aves sadias varia de 200 a $500 \mathrm{mg} / \mathrm{dL}$. Em jejum de curta duração, entre um e oito dias, a concentração sanguínea de glicose permanece notavelmente estável. Em jejum de longa duração, a glicemia é mais estável em aves carnívoras, comparadas a aves granívoras. Durante o jejum a maior perda de energia correlaciona-se à depleção de gordura e mobilização de proteínas, resultando em diminuição do peso corporal, evidenciada pela redução da massa do músculo peitoral (Thrall 2015). No presente trabalho o valor da glicemia aproximou-se mais do apresentado por Almeida et al. (2007) do que do apresentado por Medeiros et al. (2011), possivelmente devido ao fato de que os pinguins do presente trabalho estavam em adiantado estágio de reabilitação, com muitos dias de cuidados em cativeiro, em condições fisiológicas mais semelhantes às de pinguins mantidos em zoológico. De fato, Medeiros et al. (2011) relatou que os pinguins examinados em seu estudo também tinham poucos meses de reabilitação, mas que o grupo era heterogêneo quanto ao estágio da reabilitação. Coraiola (2012) relata média de glicemia semelhante à encontrada no presente trabalho.

O presente trabalho contribui para a utilização dos exames laboratoriais em pinguim-de-Magalhães, uma vez que a literatura ainda não apresenta valores de referência bem definidos para a espécie no Brasil. Entretanto, deve-se ter em consideração que as aves utilizadas no presente estudo, apesar de estarem em boas condições físicas no momento das coletas de 
sangue, foram resgatadas em estado de debilidade e estavam em um processo contínuo de recuperação da homeostasia, que certamente tem influência nos valores obtidos. Além disso, foram avaliados pinguins juvenis, e é possível que existam diferenças relevantes de parâmetros hematológicos entre as diferentes faixas etárias de pinguins-de-magalhães.

Agradecimentos.- Aos Núcleos de Fauna do IEMA e do IBAMA pelo apoio logístico e a todos os estudantes voluntários envolvidos na cadeia de atendimento aos pinguins através do IPRAM. À Fundação de Amparo à Pesquisa do Espírito Santo (FAPES) pelas bolsas concedidas. Aos alunos, estagiários e funcionários do Laboratório Clínico do Hospital Veterinário Prof. Ricardo Alexandre Hippler da UVV, especialmente Marina Drago Marchesi e Maria Fernanda Naegeli Gondim.

\section{REFERÊNCIAS}

Almeida F.M., Fedullo L.P.L., Trinta A.F., Remy G.L., Ramos Junior V.A. \& Labarthe N. 2007. Avaliação da glicose sérica em pingüim de magalhães (Spheniscus magellanicus Foster, 1781) (Sphenicidae - aves) em cativeiro. Acta Scient. Vet. 35:390-391.

Barbosa M.H. 2003. Sistema Diagnóstico de Vertebrados Superiores - SIDIVES: uma ferramenta na avaliação do impacto de óleo e derivados sobre os vertebrados superiores da Lagoa dos Patos (RS) e região costeira adjacente. Trabalho de Conclusão de Curso em Oceanologia, Fundação Universidade Federal do Rio Grande, Rio Grande, RS. 61p.

BirdLife International 2012. Spheniscus magellanicus. The IUCN Red List of Threatened Species. Version 2014.1. <www.iucnredlist.org> Downloaded on 19 July 2014.

Boersma P.D. 2008. Penguins as marine sentinels. Bioscience 58:597-607.

Campos S.D.E., Sóccio J.M.D., Oliveira R.R.G.C., Velho P.B., Maia A.L.P.S., Pereira A.M. \& Almosny N.R.P. 2008. Avaliação hematológica de 9 pingüins-de-magalhães (Spheniscus magellanicus, Foster 1781) mantidos em cativeiro. Anais do 35ํ Congresso Brasileiro de Medicina Veterinária, Gramado, RS. (Resumo)

Campos S.D.E. 2011. Estudo clínico e laboratorial da infecção por Plasmodium spp. e parasitos gastrointestinais em pinguins-de-Magalhães (Spheniscus magellanicus Foster, 1781) resgatados por três instituições no litoral sudeste do Brasil. Dissertação de Mestrado em Clínica e Reprodução Animal, Universidade Federal Fluminense, Niterói, RJ. $101 \mathrm{p}$.

Coraiola A.M. 2012. Indicadores clínicos, hematológicos, bioquímicos e toxicológicos na pré e pós-reabilitação de pinguins-de-magalhães (Spheniscus magellanicus) no sul do Brasil. Dissertação de Mestrado em Ciências Veterinárias, Universidade Federal do Paraná, Curitiba, PR. 98p.

Del Hoyo J., Elliott A. \& Sargatal J. 1992. Handbook of the birds of the world. Lynx Edicions, Spain. 696p.

Fowler M. \& Cubas Z. 2001. Biology, Medicine and Surgery of South American Wild Animals. Iowa State University Press, Ames. 550p.

Frere E., Gandini P. \& Lichtschein V. 1996. Variación latitudional em la dieta del pinguino de magallanes (Spheniscus magellanicus) em la costa patagónica, Argentina. Ornitología Neotropical 7:35-41.

García Borboroglu P., Boersma P.D., Ruoppolo V., Reyes L., Rebstock G.A., Griot K., Heredia S.R., Adornes A.C. \& Silva-Filho R.P. 2006. Chronic oil pollution harms Magellanic penguins in the Southwest Atlantic. Mar. Pollut. Bul. 52:193-198.

García Borboroglu P., Boersma P.D., Ruoppolo V., Silva Filho R.P., Adornes A.C., Sena D.C., Velozo R., Kolesnikovas C.M., Dutra G., Maracini P., Nasci- mento C.C., Ramos Junior V. \& Serra S. 2010. Magellanic penguin mortality in 2008 along the SW Atlantic coast. Mar. Pollut. Bul. 60:1652-1657.

Mäder A., Sander M. \& Casa Junior G. 2010. Ciclo sazonal de mortalidade do pinguim-de-magalhães, Spheniscus magellanicus influenciado por fatores antrópicos e climáticos na costa do Rio Grande do Sul, Brasil. Revta Bras. Ornitol. 18(3):228-233.

Medeiros L.C.C., Mayorga L.F.S.P., Bhering R.C.C., Chiparri-Gomes A.R. \& Rangel M.C.V. 2011. Avaliação da glicose sérica de pinguins de Magalhães (Spheniscus magellanicus Foster, 1781) no Espírito Santo, Brasil. Medvep - Revta Cient. Med. Vet. 36:200-202.

Moreno J., Yorio P., García-Borboroglu P., Potti J. \& Villar S. 2002. Health state and reproductive output in Magellanic penguins (Spheniscus magellanicus). Ethol. Ecol. Evolution 14:19-28.

Parsons N.J., Schaefer A.M., Spuy S.D. \& Gous T.A. 2015. Establishment of baseline haematology and biochemistry parameters in wild adult African penguins (Spheniscus demersus). J S Afr Vet Assoc. 86(1), Art. \#1198, 8 pages. DOI: $10.4102 /$ jsava.v86i1.1198

Petry M.V. \& Fonseca V.S. 2002. Effects of human activities in the marine enviroment on seabirds along the coast of Rio Grande do Sul, Brazil. Ornitología Neotropical 13:137-142.

Pinto M.B.L.C., Siciliano S. \& Di Beneditto A.P.M. 2007. Stomach contents of hte Magellanic Penguin Spheniscus magellanicus from the northern distribution limit on the Atlantic coast of Brazil. Mar. Ornithol. 35:77-78.

Pontes L.A.E., Petrucci M.P., Barbosa L.A., Ferreira F.S., Silveira L.S. \& Vieira-Da-Motta 0. 2007. Aspergilose e candidíase em pingüins de magalhães (Spheniscus magellanicus Foster, 1781) resgatados na costa do Espírito Santo, Brasil. Revta Univ. Rural, Sér. Ciências da Vida 27:57-59.

Pütz K., Ingham R.J. \& Smith J.G. 2000. Satellite tracking of the winter migration of Magellanic penguins (Spheniscus magellanicus) breeding in the Falkland Islands. Ibis 142(4):614-622.

Pütz K., Ingham R.J. \& Smith J.G. 2007. Winter migration of Magellanic penguins (Spheniscus magellanicus) from the southernmost distributional range. Mar. Biol. 152:1227-1235.

Rajman M., Juráni M., Lamosova D., Macajova M., Sedlackova M., Kostal L., Jezova D. \& Vyboh P. 2006. The effects of feed restriction on plasma biochemistry in growing meat type chickens (Gallus gallus). Comp. Biochem. Physiol. 145(3):363-371.

Rodrigues S.C., Adornes A.C., Santos Filho E.A., Silva Filho R.P., Colares E.P. 2010. Surviving probability indicators of landing juvenile Magellanic Penguins arriving along the Southern Brazilian coast. Braz. Arch. Biol. Technol. 53(2):419-424.

Sick H. 1997. Ornitologia Brasileira: uma introdução. Nova Fronteira, Rio de Janeiro. 912p.

Silva N.W.X., Vargas M.P., Zanini S.F., Almeida A.T.S., Carvalho M.A.G. \& Almada G.L. 2001. Levantamento preliminar de pinguins de magalhães (Spheniscus magellanicus) (Sphenisciformes) reabilitados na costa capixaba. Anais 5은 Congresso ABRAVAS e $10^{\circ}$ Encontro ABRAVAS, São Paulo, SP. (Resumo)

Silva Filho R.P. \& Ruoppolo V. 2015. Sphenisciformes (Pinguim), p.384416. In: Cubas Z.S., Silva J.C. \& Catão-Dias J.L. (Eds), Tratado de Animais Selvagens: medicina veterinária. $2^{\mathrm{a}}$ ed. Roca, São Paulo.

Stokes D.L., Boersma P.D. \& Davis L.S. 1998. Satellite tracking of Magellanic penguins migration. Condor 100:376-381.

Thrall M.A. 2015. Hematologia e Bioquímica Clínica Veterinária. 2a ed. Roca, São Paulo, 678p.

Villouta G., Hargreaves R. \& Rtveros V. 1997. Haematological and clinical biochemistry findings in captive Humboldt penguins (Spheniscus humboldti). Avian Pathol. 26(4):851-858.

Williams T.D. 1995. The Penguins. Oxford University Press, Oxford. 295p. 